\title{
The relationship between moderate-to-vigorous physical activity and executive function among individuals with schizophrenia: differences by illness duration
}

\author{
Viviane Grassmann, ${ }^{1}$ Mehala Subramaniapillai, ${ }^{1}$ Mark Duncan, ${ }^{2}$ Kelly Arbour-Nicitopoulos, ${ }^{1}$ \\ Guy E. Faulkner \\ ${ }^{1}$ University of Toronto, Toronto, ON, Canada. ${ }^{2}$ University of British Columbia, Vancouver, BC, Canada.
}

\begin{abstract}
Objective: Schizophrenia is a chronic mental illness characterized by positive and negative symptoms. Cognitive impairment continues to be a core and consistent deficit. Previous studies have shown that physical activity (PA) is positively associated with cognitive performance. Thus, it may play a supportive role in mitigating cognitive impairments among individuals with schizophrenia. The aim of this study was to analyze the relationship between moderate-to-vigorous physical activity (MVPA) and executive function among adults with schizophrenia.

Methods: The weekly amount of MVPA (assessed using accelerometers) and executive function (as per Brief Neurocognitive Assessment for Schizophrenia) of 78 adults with schizophrenia (mean [SD] age 42.4 [11.4] years; illness duration 17.0 [11.0] years; $58.2 \%$ male) were assessed in this crosssectional study. Pearson correlations were calculated, followed by a linear regression. Participants were first analyzed together and then dichotomized on the basis of illness duration.

Results: There was no significant association between MVPA and executive function, independent of the duration of illness. For individuals with $<15$ years of illness, there was a significant association between weekly MVPA and working memory performance.

Conclusion: PA appears to be associated with executive function in some, but not all, individuals with schizophrenia.
\end{abstract}

Keywords: Accelerometry; executive function; physical activity; schizophrenia

\section{Introduction}

Schizophrenia is a chronic mental illness characterized by disorders of perception and thinking. ${ }^{1}$ Individuals with this illness often present positive (e.g., hallucination, delusion, and thought disorders) and negative symptoms (e.g., avolition, apathy, and social dysfunction). ${ }^{2}$ Cognitive impairment is also common, and has a significant, detrimental impact on quality of life and functional outcome. Deficits vary among individuals, but often affect multiple cognitive domains, such as attention, executive function, memory, social cognition, and language. ${ }^{3,4}$

Although antipsychotic drugs can improve many symptoms of this disease, treatment has little effect on alleviating cognitive impairment. ${ }^{5-7}$ Since cognitive function is a strong predictor of functional outcomes, ${ }^{8}$ it is critical to explore new strategies to attenuate this impairment. One such strategy may be through physical activity (PA). PA has been shown to improve cognitive function in individuals with different chronic health conditions, including dementia, attention-deficit hyperactivity disorder, and depression. ${ }^{9-11}$

Correspondence: Viviane Grassmann, Faculty of Kinesiology and Physical Education, University of Toronto, 55 Harbord St., Toronto, ON Canada M5S 2W6.

E-mail: viviane.grassmann @utoronto.ca

Submitted Sep 11 2016, accepted Dec 03 2016, Epub Mar 302017.
Some studies have suggested that even low doses of moderate-to-vigorous physical activity (MVPA), e.g., 10-minute bouts, can have a positive influence on cognitive function. ${ }^{12,13}$

Studies conducted with healthy adults show a positive association between PA and executive function (namely, attention control and working memory). ${ }^{14-16}$ These benefits are postulated to be influenced by increases in neurotrophic factors (e.g., brain-derived neurotrophic factor and insulin growth factor 1), in brain volume, and/or cerebral blood flow. ${ }^{17,18}$ This association between PA and executive function also seems to hold true among individuals with schizophrenia. ${ }^{19-22}$ In a recent meta-analysis, Firth et al. ${ }^{22}$ demonstrated that PA interventions can improve global cognition and several sub-domains of cognition (working memory, social cognition, and attention/vigilance). Cross-sectional studies have also demonstrated a relationship of PA and sedentary behavior with cognitive function. ${ }^{19-21,23}$ A recent study demonstrated an independent and negative association between cognitive performance and sedentary behavior and/or physical inactivity. ${ }^{21}$ However, MVPA is positively associated with cognitive performance. ${ }^{19,20}$ Leutwyler et al. ${ }^{19}$ evaluated older adults with schizophrenia using an objective measure of MVPA and demonstrated that speed of processing (category fluency) and working memory (assessed with a letter-numbers span task) were positively associated with MVPA. However, the 
authors did not control for potential confounders, such as demographic factors (e.g., level of education) and clinical features (e.g., years of illness), that may explain the association between PA and executive functioning. Chen et al. ${ }^{20}$ conducted a similar study with adult inpatients and controlled for some demographic and clinical variables, including age, body mass index (BMI), metabolic parameters, and medication use. They reported a positive association between attention and concentration (assessed with the Cognitrone test) and speed of processing (motor process/grooved pegboard test) and MVPA. Given the limited extent of existing research and the inconsistency in reported findings, further investigations are warranted to explore the potential role of PA in moderating the common cognitive dysfunction seen in individuals with schizophrenia.

Within this context, the aim of this study was to analyze the relationship between weekly minutes engaged in MVPA and executive function among adults with schizophrenia, controlling for potential demographic and clinical confounders. We hypothesized that higher levels of MVPA would be associated with superior performance on cognitive function tasks, while taking into account that such an association might vary with illness duration, as previous studies have shown that, although cognitive function deteriorates gradually through the course of illness, this process is not linear. ${ }^{24-26}$

\section{Methods}

\section{Participants}

Individuals with schizophrenia were recruited from the Centre for Addiction and Mental Health (CAMH), Toronto, Canada. The following inclusion criteria were established: 1) adults aged 18-64 years (in line with the age range of $P A$ guidelines for adults in Canada); 2) inpatients and outpatients diagnosed with schizophrenia, schizoaffective disorder, or psychosis not-otherwise-specified (NOS); and 3 ) no changes in antipsychotic treatment in the preceding 4 weeks. Participants were excluded if: 1) they had been hospitalized in the past 12 months for angina pectoris, myocardial infarction, or cardiac surgery of any kind; or 2) had uncontrolled hypertension (defined as blood pressure $>140 / 90 \mathrm{mmHg}$ ).

Overall, 140 participants enrolled in the study, of whom 62 were excluded due to: failure to complete the executive function task $(n=45)$, incomplete accelerometry data $(n=11)$, or missing data related to their disease (illness duration, $\mathrm{n}=1$; antipsychotic medication, $\mathrm{n}=4$ ). One more participant was excluded due to being an outlier on the executive function performance task $(n=1)$. This reduced the final sample size to 78 . There were no differences between the excluded participants and those included in any variables except age and sex: those excluded were younger (37.25 $11.01)$ and were more likely to be male $(87.5 \%)$.

\section{Procedures}

Data collection began in May 2014 and concluded in April 2015. The Mini-International Neuropsychiatric Interview
$(\mathrm{MINI})^{27}$ was used to confirm diagnosis during the first session. Participants also provided basic demographic, clinical, and anthropometric information during this visit. During session two, participants completed the Symbol Coding and Letters and Numbers Span tests of the Brief Neurocognitive Assessment for Schizophrenia (BNAS) to assess executive function and were asked to wear an accelerometer for 7 consecutive days to obtain a measure of PA. This study was part of a larger project examining the determinants of PA in this population. ${ }^{28}$ Ethics approval was received from CAMH and the University of Toronto Research Ethics Boards. Participants had their capacity to consent evaluated at the beginning of the study using the MacArthur Competence Assessment Tool for Clinical Research (MacCAT-CR) ${ }^{29}$ Participants were included only if they were considered able to provide written consent.

\section{Measures}

\section{Executive function/attention}

Executive function was evaluated using the BNAS, ${ }^{30}$ which includes two tasks. In the first task (Letters and Numbers Span task) (University of Maryland), participants were asked to reorder intermixed sequences of letters and numbers. ${ }^{31}$ In the second, the Symbol Coding task, participants were asked to transcribe numbers to their corresponding symbols to test speed processing and attention. ${ }^{7}$ Previous research has shown the BNAS to be a reliable and valid neurocognitive assessment for patients with schizophrenia. ${ }^{30}$

\section{Physical activity (PA)}

PA was measured using an Actigraph wGT3X+ accelerometer. Participants were instructed to wear the device on their right hip for 7 consecutive days. Accelerometry data were analyzed using the Actigraph Actilife program (v6.12). Wear time was validated using the algorithm developed by Choi et al. ${ }^{32} \mathrm{~A}$ valid wear day was considered to be 600 minutes of wear time, while a valid week was defined at least 4 valid days within the 7-day period. ${ }^{33,34}$ The PA cutoffs established by Troiano et al. ${ }^{34}$ were used to determine time spent in MVPA ( $\geqslant 2,020$ counts per minute), with only bouts of MVPA lasting at least 10 minutes being included, as per PA guidelines. ${ }^{35}$ Weekly MVPA was then calculated using the average daily MVPA among valid wear days, multiplied across the 7 days. According to PA guidelines, adults should participate in at least 150 minutes of MVPA per week. ${ }^{35}$ Thus, the sample was categorized into two levels for descriptive analysis: those who met PA guidelines and those who did not.

\section{Covariates}

A range of covariates was assessed. These included medication dosage (chlorpromazine equivalents), illness duration, and educational attainment.

\section{Chlorpromazine equivalents (CPZ)}

As studies have shown that some antipsychotics could modestly improve cognitive function performance, ${ }^{5,6}$ it is 
important to include antipsychotic dosage in analyses. CPZ has clinical and research applications by estimating the dose equivalence across different antipsychotic medications. For this study, each participant's current antipsychotic dosage was calculated as per Gardner et al. ${ }^{36}$

\section{IIIness duration}

Considering that the relationship between cognitive function and illness duration may not be linear, ${ }^{24-26}$ and that illness duration is used as an approximation to the stage of illness, ${ }^{25}$ the sample was dichotomized on the basis of illness duration using a median split (median $=14.17$ years; first quartile $=9.42$; third quartile $=24.33$ years; $\leqslant 15$ years $[n=44]$ vs. $>15$ years $[n=34]$ )

\section{Level of education}

Educational attainment has been consistently shown to be associated with cognitive function. ${ }^{37,38}$ This variable was dichotomized into two categories: a) lower education (participants without a high school diploma); and b) higher education (those with a high school diploma).

\section{Data analyses}

All analyses were conducted using SPSS version 23. Data cleaning procedures were conducted to examine univariate outliers and normality. ${ }^{39}$ Main analyses were first conducted on all participants together, followed by a dichotomized analysis. Differences in demographic and clinical variables, MVPA, and executive function between the two dichotomized illness groups ( $\leqslant 15$ and $>15$ years of illness) were compared using an independent-sample $t$-test or chi-square analyses as appropriate. The relationships between MVPA, CPZ, and executive function were analyzed using Pearson's correlations. Variables that showed a significant $(p<0.05)$ correlation were included in the linear regression models. Two regression models were constructed for all participants, and then for the dichotomized illness groups ( $\leqslant 15$ and $>15$ years of illness). In the first model, the Symbol Coding task was used to predict weekly MVPA, while the second model used the Letters and Numbers Span task as a predictor of weekly MVPA (controlling for significant demographic variables). Significance was set at $p<0.05$ in all analyses.

\section{Results}

\section{Group differences}

Descriptive characteristics of the sample are presented in Table 1, for all participants and stratified by years of illness ( $\leqslant 15-$ Yol and $>15-$ Yol). As expected, between-group, independent $t$-tests revealed that individuals in the $>15$-Yol group were older $(t=-8.66, \mathrm{p}<0.001)$ than individuals in the $\leqslant 15$-Yol group. No other significant differences between these groups emerged on any other demographic or anthropometric variables, such as BMI, CPZ, gender, smoking habits, and education ( $p>0.05)$.

There was a higher proportion of individuals with schizophrenia in the $\leqslant 15$-Yol group, while the $>15$-Yol group showed a higher proportion of individuals with a diagnosis of schizoaffective disorder $\left(\chi^{2}=6.59, p=0.04\right)$. No significant between-group differences were found in total time spent in MVPA per week $(t=1.78, p>0.05)$. However, a higher proportion of participants in the $\leqslant 15$ Yol group met the PA guidelines of 150 minutes of MVPA per week $\left(\chi^{2}=4.89, p=0.03\right.$; $>150$ minutes of PA per week) compared to those in the $>15$-Yol group. Furthermore, individuals in the $\leqslant 15$-Yol group performed significantly better on the Symbol Coding task $(t=-5.45$, $p<0.001)$ when compared to those individuals in the $>15$-Yol group. However, there was no difference between these groups on the Letters and Numbers Task $(t=-1.55$, $p>0.05)$.

Associations between executive functioning and moderateto-vigorous physical activity (MVPA) and chlorpromazine equivalents $(C P Z)$ in the sample as a whole

Table 2 presents the correlations between performance on the executive function tasks, MVPA, and CPZ. CPZ correlated negatively with assessments of executive function. There were also significant correlations between illness duration and performance on the Symbol Coding task $(r=-0.52, p=<0.001)$, total time spent in MVPA per week $(r=-0.32, \mathrm{p}=0.005)$, and CPZ $(r=0.26, \mathrm{p}=0.024)$. Linear regression identified that $\mathrm{CPZ}$ and illness duration were the only significant predictors of performance in the Symbol Coding task (Table 3); MVPA and level of education were not significant predictors. Illness duration was, however, a significant predictor of MVPA $(\beta=$ -0.32, $t=-2.92, \mathrm{p}=0.005 ;$ adj. $\mathrm{R}^{2}=0.09, \mathrm{~F}_{1,77}=8.55$, $\mathrm{p}=0.005)$.

Associations between executive functioning and moderateto-vigorous physical activity (MVPA) and chlorpromazine equivalents (CPZ) after dichotomization of illness duration ( $\leqslant 15$ year of illness vs. > 15 year of illness)

There was no association between MVPA and executive function among individuals with more than 15 years of illness (Table 2). However, among participants with 15 or fewer years of illness, MVPA predicted performance on the Letters and Numbers Span task, but not on the Symbol Coding task, even when controlling for CPZ and educational level in the model (Table 4).

\section{Discussion}

Overall, this study demonstrated no relationship between MVPA and executive function in a general sample of individuals with schizophrenia or schizoaffective disorder. However, analyses did find an association between MVPA and performance on a working memory task for individuals with 15 or fewer years of illness. Among all participants, duration of illness was a predictor of MVPA, which supports Vancampfort's finding of an association between physical fitness and illness duration that was independent of age. ${ }^{40}$ Moreover, in our results, illness duration and dose of antipsychotics were also predictors of speed of processing and attention performance, as assessed by the Symbol 
Table 1 Descriptive statistics and differences between groups

\begin{tabular}{|c|c|c|c|}
\hline & All participants $(n=78)$ & $\leqslant 15$ years of illness $(n=44)$ & $>15$ years of illness $(n=34)$ \\
\hline Age (years), mean (SD) & $42.39(11.44)$ & $35.27(8.92)$ & $51.34(7.17)^{*}$ \\
\hline Gender (male) & $46(58.2)$ & $25(56.8)$ & $21(60.0)$ \\
\hline Smoking (nonsmoker or past smoker) & $43(54.4)$ & $24(54.5)$ & $19(54.3)$ \\
\hline BMI $\left(\mathrm{kg} / \mathrm{m}^{2}\right)$, mean (SD) & $31.67(8.04)$ & $32.60(9.14)$ & $30.51(6.32)$ \\
\hline Illness duration (years), mean (SD) & 17.06 (10.99) & $9.17(4.10)$ & $26.73(8.40)^{*}$ \\
\hline CPZ (mg/day), mean (SD) & $559.19(394.08)$ & $521.73(312.98)$ & $606.29(477.80)$ \\
\hline \multicolumn{4}{|l|}{ Diagnosis $^{\dagger}$} \\
\hline Schizophrenia & $48(60.8)$ & $32(72.7)$ & $16(45.7)$ \\
\hline Schizoaffective disorder & $30(38.0)$ & $12(27.3)$ & $18(51.4)$ \\
\hline Psychosis NOS & $1(1.3)$ & $0(0.0)$ & $1(2.9)$ \\
\hline \multicolumn{4}{|l|}{ Education } \\
\hline High school diploma & $64(81)$ & $37(84.1)$ & $27(77.1)$ \\
\hline No high school diploma & $14(17.7)$ & $6(13.6)$ & $8(22.9)$ \\
\hline Other & $1(1.3)$ & $1(2.3)$ & $0(0.0)$ \\
\hline \multicolumn{4}{|l|}{ Executive function, mean (SD) } \\
\hline Symbol Coding & $44.03(12.33)$ & $49.88(12.05)$ & $36.83(8.25)^{*}$ \\
\hline Letters and Numbers Span & $13.13(3.47)$ & $13.66(3.23)$ & $12.44(3.70)$ \\
\hline \multicolumn{4}{|l|}{ Met PA guidelines ${ }^{\dagger}$} \\
\hline Yes & $48(60.8)$ & $32(72.7)$ & $16(45.7)$ \\
\hline No & $31(39.2)$ & $12(27.3)$ & $19(54.3)$ \\
\hline \multicolumn{4}{|l|}{ MVPA, mean (SD) } \\
\hline Total MVPA (minutes/week) & $252.77(206.61)$ & $289.26(197.71)$ & $206.90(211.20)$ \\
\hline MVPA time per bout (minutes) & $15.04(5.02)$ & $15.85(5.87)$ & $14.02(3.49)$ \\
\hline
\end{tabular}

Data presented as $\mathrm{n}(\%)$, unless otherwise specified.

$\mathrm{CPZ}=$ chlorpromazine equivalents; MVPA = moderate-to-vigorous physical activity; NOS = not otherwise specified; $\mathrm{SD}=\mathrm{standard}$ deviation

$* p<0.001$ and ${ }^{\dagger} p<0.05$ for differences between $\leqslant 15$ years of illness and $>15$ years of illness.

Table 2 Correlation between executive function, MVPA, and chlorpromazine equivalents

\begin{tabular}{|c|c|c|c|c|}
\hline & \multicolumn{2}{|c|}{ Executive function } & \multirow[b]{2}{*}{ MVPA } & \multirow[b]{2}{*}{$\mathrm{CPZ}$} \\
\hline & Symbol Coding & Letters and Numbers Span & & \\
\hline \multicolumn{5}{|l|}{ All participants } \\
\hline \multicolumn{5}{|l|}{ Executive function } \\
\hline Symbol Coding & - & & & \\
\hline Letters and Numbers Span & $0.54 *$ & - & & \\
\hline MVPA & 0.17 & 0.09 & - & \\
\hline $\mathrm{CPZ}$ & $-0.34 *$ & $-0.23^{\dagger}$ & -0.03 & - \\
\hline \multicolumn{5}{|l|}{$\leqslant 15$ years of illness } \\
\hline \multicolumn{5}{|l|}{ Executive function } \\
\hline Symbol Coding & - & & & \\
\hline Letters and Numbers Span & $0.57 *$ & - & & \\
\hline MVPA & 0.17 & $0.34^{\dagger}$ & - & \\
\hline $\mathrm{CPZ}$ & $-0.47^{*}$ & -0.29 & -0.18 & - \\
\hline \multicolumn{5}{|l|}{$>15$ years of illness } \\
\hline \multicolumn{5}{|l|}{ Executive function } \\
\hline Symbol Coding & - & & & \\
\hline Letters and Numbers Span & $0.48^{*}$ & - & & \\
\hline MVPA & -0.02 & -0.22 & - & \\
\hline $\mathrm{CPZ}$ & -0.22 & -0.16 & 0.107 & - \\
\hline
\end{tabular}

$\mathrm{CPZ}=$ chlorpromazine equivalents; MVPA = moderate-to-vigorous physical activity (minutes per week).

$*<0.01 ; \dagger p<0.05$.

Coding task. No association was shown between executive functioning and MVPA among those with more than 15 years of illness. This null result could be due to the chronicity of schizophrenia, particularly considering that these participants had demonstrated greater cognitive impairment (based on the Symbol Coding task), which is consistent with previous literature. ${ }^{25,26}$ Duration of illness appears to be an important factor to be considered further when exploring the relationship between PA (and MVPA in particular) and cognitive function. 
Table 3 Linear regression predicting executive function task performance for all participants

\begin{tabular}{|c|c|c|c|c|c|c|c|c|}
\hline \multirow[b]{3}{*}{ Predictors } & \multicolumn{8}{|c|}{ All participants } \\
\hline & \multicolumn{4}{|c|}{ Symbol Coding } & \multicolumn{4}{|c|}{ Letters and Numbers Span } \\
\hline & Adjusted $\mathrm{R}^{2}$ & $F$ for $R^{2}$ & $\beta$ & $t$ & Adjusted $\mathrm{R}^{2}$ & $F$ for $R^{2}$ & $\beta$ & $t$ \\
\hline & 0.32 & $12.71 *$ & & & 0.04 & 2.06 & & \\
\hline $\mathrm{CPZ}$ & & & -0.21 & $-2.15^{\dagger}$ & & & -0.21 & -1.81 \\
\hline Illness duration & & & -0.43 & $-4.25^{*}$ & & & -0.09 & -0.76 \\
\hline Educational level & & & 0.16 & 1.59 & & & 0.08 & 0.75 \\
\hline
\end{tabular}

$\mathrm{CPZ}=$ chlorpromazine equivalents; MVPA = moderate-to-vigorous physical activity.

$*<0.001 ;{ }^{\dagger} \mathrm{p}<0.05$.

Table 4 Linear regression predicting executive function task performance for the participants with $\leqslant 15$ years of illness

$\leqslant 15$ years of illness

\begin{tabular}{|c|c|c|c|c|c|c|c|c|}
\hline \multirow[b]{2}{*}{ Step/predictors } & \multicolumn{4}{|c|}{ Symbol Coding } & \multicolumn{4}{|c|}{ Letters and Numbers Span } \\
\hline & Adjusted $\mathrm{R}^{2}$ & $F$ for $R^{2}$ & $\beta$ & $t$ & Adjusted $\mathrm{R}^{2}$ & $F$ for $R^{2}$ & $\beta$ & $t$ \\
\hline Step 1 & 0.01 & 1.24 & & & 0.11 & $6.14^{*}$ & & \\
\hline MVPA & & & 0.17 & 1.11 & & & 0.36 & $2.48 *$ \\
\hline Step 2 & 0.19 & $4.29 *$ & & & 0.13 & $3.11^{*}$ & & \\
\hline MVPA & & & 0.05 & 0.32 & & & 0.32 & $2.09 *$ \\
\hline $\mathrm{CPZ}$ & & & -0.45 & $-3.16^{\dagger}$ & & & -0.26 & -1.74 \\
\hline Educational level & & & 0.13 & 0.39 & & & -0.02 & -0.14 \\
\hline
\end{tabular}

$\mathrm{CPZ}=$ chlorpromazine equivalents; MVPA = moderate-to-vigorous physical activity (minutes per week); Step $1=$ linear regression between MVPA and executive function; Step 2 = linear regression between MVPA and executive function including demographical and clinical confounders.

$* p<0.05 ;{ }^{\dagger}<0.01$

For individuals with 15 or fewer years of illness, there was also no correlation between MVPA and speed of processing/attention. This null result is in line with the findings from a recent systematic review in a healthy population showing a small to no effect of PA level on the speed of processing. ${ }^{14}$ Although previous studies ${ }^{19,20}$ with individuals with schizophrenia found a positive correlation between MVPA and speed of processing/attention, these findings should be treated with caution, as one study did not control for potential confounders and both assessed cognitive functioning differently. ${ }^{19,20}$ Stubbs et al. ${ }^{21}$ demonstrated a negative association between speed of processing/attention and sedentary behavior (as well as physical inactivity); however, regarding PA specifically, a study by Kramer et al. $^{41}$ showed that it is selectively related to tasks that require more extensive amounts of executive control. The working memory test used in the present study (i.e., the Letters and Numbers Span task) requires more executive control than the Symbol Coding task, and demonstrated a significant relationship with MVPA for those with 15 or fewer years of illness. This relationship remained when controlling for medication dosage. Thus, in our sample, the relationship between working memory and MVPA corroborates with previous cross-sectional and intervention studies evaluating individuals with ${ }^{19}$ and without schizophrenia. ${ }^{15,16}$ The present results extend the findings of Leutwyler et al. ${ }^{19}$ by demonstrating a relationship between working memory and MVPA even when controlling for medication dosage and level of education (for those with 15 or fewer years of illness). This relationship could be due to an increase in cerebral blood flow, neurotrophic factors, or even to an increase in brain volume. ${ }^{17,18,42,43}$
In brief, our results show that a higher level of MVPA is associated with better performance on a working memory task for those with 15 or fewer years of schizophrenia. Future experimental research should examine whether PA-mediated improvements in cognitive function have any clinical benefit.

There are some limitations in this study that need to be considered. First, we were unable to control for other potential confounders (e.g., smoking or BMI) due to the small sample size. Considering previous literature has shown an association between obesity and cognitive function, ${ }^{44,45}$ we also conducted additional analyses (data not shown) and found no association between cognitive function and BMI $(p>0.05)$ in this sample. Second, information about medical comorbidities was not collected. Future studies could control for these, especially those comorbidities that influence cognitive function (e.g., diabetes or respiratory diseases). Third, although accelerometers are accurate in assessing the volume of PA, they cannot determine which type of activity that participants engaged in. Type of activity might be an important moderator of the PA and cognitive functioning relationship. Fourth, the BNAS evaluates only some aspects of cognitive function. It is possible that the time spent in MVPA could be related to long-term memory or other aspects of executive function (e.g., inhibition, problem solving, and language) not assessed by the BNAS. Finally, the cross-sectional design of the study prevents any determination of causality and directionality. Previous research by Stubbs has shown that cognitive deficit is one of the key factors explaining the reduced amount of PA among individuals with schizophrenia. ${ }^{23}$ 
In conclusion, PA does not appear to be associated with executive function for all individuals with schizophrenia. However, individuals with 15 or fewer years of illness who engaged in greater MVPA during the week of assessment performed better in terms of working memory. Further investigation is required to elucidate the potential role of PA in alleviating the cognitive dysfunction commonly experienced by individuals with schizophrenia.

In terms of clinical implications, it is known that PA is important to physical and mental health in this population. ${ }^{22,46}$ Cognitive deficits are common in schizophrenia, and PA may also play a role in attenuating such deficits. Therefore, health care professionals should be informed about these benefits and help patients increase PA. ${ }^{22,23}$ However, due to cognitive limitations, individuals with schizophrenia may require long-term support to maintain engagement in PA. ${ }^{47}$

\section{Acknowledgements}

This work was supported by a Canadian Institutes of Health Research operating grant (PI: GEF). VG received financial support from Conselho Nacional de Desenvolvimento Científico e Tecnológico (CNPq; grant no. 202987/ 2014-0). These funding sources had no further role in the study design, statistical analysis, and interpretation of findings, writing of the manuscript or in the decision to submit for publication. The authors thank the Centre for Addiction and Mental Health staff and the participants for volunteering their time for this study.

\section{Disclosure}

The authors report no conflicts of interest.

\section{References}

1 Balhara YP, Verma R. Schizophrenia and suicide. East Asian Arch Psychiatry. 2012;22:126-33.

2 American Psychiatric Association. Diagnostic and Statistical Manual of Mental Disorders, Fifth Edition (DSM-5). Arlington: American Psychiatric Publishing; 2013.

3 Kalkstein S, Hurford I, Gur RC. Neurocognition in schizophrenia. Curr Top Behav Neurosci. 2010;4:373-90.

4 Millan MJ, Agid Y, Brune M, Bullmore ET, Carter CS, Clayton NS, et al. Cognitive dysfunction in psychiatric disorders: characteristics, causes and the quest for improved therapy. Nat Rev Drug Discov. 2012;11:141-68.

5 Breier A. Developing drugs for cognitive impairment in schizophrenia. Schizophr Bull. 2005;31:816-22.

6 Harvey PD, Keefe RS. Studies of cognitive change in patients with schizophrenia following novel antipsychotic treatment. Am J Psychiatry. 2001;158:176-84.

7 Keefe RS, Silva SG, Perkins DO, Lieberman JA. The effects of atypical antipsychotic drugs on neurocognitive impairment in schizophrenia: a review and meta-analysis. Schizophr Bull. 1999;25:201-22.

8 Green MF, Kern RS, Braff DL, Mintz J. Neurocognitive deficits and functional outcome in schizophrenia: are we measuring the "right stuff"? Schizophr Bull. 2000;26:119-36.

9 Grassmann V, Alves MV, Santos-Galduroz RF, Galduroz JC. Possible cognitive benefits of acute physical exercise in children with ADHD: a systematic review. J Atten Disord. 2017;21:367-71.

10 Blondell SJ, Hammersley-Mather R, Veerman JL. Does physical activity prevent cognitive decline and dementia?: A systematic review and meta-analysis of longitudinal studies. BMC Public Health. 2014; $14: 510$
11 Mammen G, Faulkner G. Physical activity and the prevention of depression: a systematic review of prospective studies. Am J Prev Med. 2013;45:649-57.

12 Marinac CR, Godbole S, Kerr J, Natarajan L, Patterson RE, Hartman SJ. Objectively measured physical activity and cognitive functioning in breast cancer survivors. J Cancer Surviv. 2015;9:230-8.

13 Vidoni ED, Johnson DK, Morris JK, Van Sciver A, Greer CS, Billinger $\mathrm{SA}$, et al. Dose-response of aerobic exercise on cognition: a community-based, pilot randomized controlled trial. PLoS One. 2015;10: e0131647.

14 Cox EP, O'Dwyer N, Cook R, Vetter M, Cheng HL, Rooney K, et al. Relationship between physical activity and cognitive function in apparently healthy young to middle-aged adults: a systematic review. J Sci Med Sport. 2016;19:616-28.

15 Erickson KI, Banducci SE, Weinstein AM, Macdonald AW 3rd, Ferrell $\mathrm{RE}$, Halder I, et al. The brain-derived neurotrophic factor Val66Met polymorphism moderates an effect of physical activity on working memory performance. Psychol Sci. 2013;24:1770-9.

16 Padilla C, Perez L, Andres P. Chronic exercise keeps working memory and inhibitory capacities fit. Front Behav Neurosci. 2014;8: 49-49.

17 Phillips C, Baktir MA, Srivatsan M, Salehi A. Neuroprotective effects of physical activity on the brain: a closer look at trophic factor signaling. Front Cell Neurosci. 2014;8:170.

18 Cotman CW, Berchtold NC. Exercise: a behavioral intervention to enhance brain health and plasticity. Trends Neurosci. 2002;25: 295-301.

19 Leutwyler H, Hubbard EM, Jeste DV, Miller B, Vinogradov S. Associations of schizophrenia symptoms and neurocognition with physical activity in older adults with schizophrenia. Biol Res Nurs. 2014;16: 23-30.

20 Chen LJ, Steptoe A, Chung MS, Ku PW. Association between actigraphy-derived physical activity and cognitive performance in patients with schizophrenia. Psychol Med. 2016;46:2375-84.

21 Stubbs B, Ku PW, Chung MS, Chen LJ. Relationship between objectively measured sedentary behavior and cognitive performance in patients with schizophrenia vs controls. Schizophr Bull. 2016 Aug 23. pii: sbw126. [Epub ahead of print].

22 Firth J, Stubbs B, Rosenbaum S, Vancampfort D, Malchow B, Schuch $F$, et al. Aerobic exercise improves cognitive functioning in people with schizophrenia: a systematic review and metaanalysis. Schizophr Bull. 2016 Aug 11. pii: sbw115. [Epub ahead of print].

23 Stubbs B, Koyanagi A, Schuch F, Firth J, Rosenbaum S, Gaughran $F$, et al. Physical activity levels and psychosis: a mediation analysis of factors influencing physical activity target achievement among 204186 people across 46 low- and middle-income countries. Schizophr Bull. 2016 Aug 24. pii: sbw111. [Epub ahead of print].

24 van Haren NE, Cahn W, Hulshoff Pol HE, Kahn RS. Schizophrenia as a progressive brain disease. Eur Psychiatry. 2008;23:245-54.

25 Bagney A, Rodriguez-Jimenez R, Martinez-Gras I, Sanchez-Morla EM, Santos JL, Jimenez-Arriero MA, et al. Negative symptoms and executive function in schizophrenia: does their relationship change with illness duration? Psychopathology. 2013;46:241-8.

26 Shmukler AB, Gurovich IY, Agius M, Zaytseva Y. Long-term trajectories of cognitive deficits in schizophrenia: a critical overview. Eur Psychiatry. 2015;30:1002-10.

27 Sheehan DV, Lecrubier Y, Sheehan KH, Amorim P, Janavs J, Weiller E, et al. The Mini-International Neuropsychiatric Interview (M.I.N.I.): the development and validation of a structured diagnostic psychiatric interview for DSM-IV and ICD-10. J Clin Psychiatry. 1998;59: 22-33.

28 Arbour-Nicitopoulos KP, Duncan M, Remington G, Cairney J, Faulkner GE. Development and reliability testing of a health action process approach inventory for physical activity participation among individuals with schizophrenia. Front Psychiatry. 2014;5:68.

29 Appelbaum PS, Grisso T. MacArthur competence assessment tool for clinical research (MacCAT-CR)Sarasota: Professional Resource Press; 2001.

30 Fervaha G, Agid O, Foussias G, Siddiqui I, Takeuchi H, Remington G. Neurocognitive impairment in the deficit subtype of schizophrenia. Eur Arch Psychiatry Clin Neurosci. 2016;266:397-407.

31 Gold JM, Queenr C, lannone VN, Buchanan RW. Repeatable battery for the assessment of neuropsychological status as a screening test 
in schizophrenia I: sensitivity, reliability, and validity. Am J Psychiatry. 1999;156:1944-50.

32 Choi L, Liu Z, Matthews CE, Buchowski MS. Validation of accelerometer wear and nonwear time classification algorithm. Med Sci Sports Exerc. 2011;43:357-64.

33 Trost SG, Mclver KL, Pate RR. Conducting accelerometer-based activity assessments in field-based research. Med Sci Sports Exerc. 2005;37:S531-43.

34 Troiano RP, Berrigan D, Dodd KW, Masse LC, Tilert T, McDowell M. Physical activity in the United States measured by accelerometer. Med Sci Sports Exerc. 2008;40:181-8.

35 Warburton DE, Charlesworth S, Ivey A, Nettlefold L, Bredin SS A systematic review of the evidence for Canada's physical activity guidelines for adults. Int J Behav Nutr Phys Act. 2010;7:39.

36 Gardner DM, Murphy AL, O'Donnell H, Centorrino F, Baldessarini RJ. International consensus study of antipsychotic dosing. Am J Psychiatry. 2010;167:666-93.

37 Lee S, Kawachi I, Berkman LF, Grodstein F. Education, other socioeconomic indicators, and cognitive function. Am J Epidemiol. 2003;157:712-20.

38 Ho YT, Kao TW, Peng TC, Liaw FY, Yang HF, Sun YS, et al. Role of educational status in explaining the association between body mass index and cognitive function. Medicine (Baltimore). 2016;95:e2656.

39 Pallant J. SPSS survival manual. New York: Open University Press; 2013.
40 Vancampfort D, Probst M, Scheewe T, De Herdt A, Sweers K Knapen J, et al. Relationships between physical fitness, physical activity, smoking and metabolic and mental health parameters in people with schizophrenia. Psychiatry Res. 2013;207:25-32.

41 Kramer AF, Hahn S, Cohen NJ, Banich MT, McAuley E, Harrison CR, et al. Ageing, fitness and neurocognitive function. Nature. 1999;400: 418-9.

42 Cassilhas RC, Viana VA, Grassmann V, Santos RT, Santos RF, Tufik $\mathrm{S}$, et al. The impact of resistance exercise on the cognitive function of the elderly. Med Sci Sports Exerc. 2007;39:1401-7.

43 Okereke O, Kang JH, Ma J, Hankinson SE, Pollak MN, Grodstein F. Plasma IGF-I levels and cognitive performance in older women. Neurobiol Aging. 2007;28:135-42.

44 Guo X, Zhang Z, Wei Q, Lv H, Wu R, Zhao J. The relationship between obesity and neurocognitive function in Chinese patients with schizophrenia. BMC Psychiatry. 2013;13:109.

45 Rashid NA, Lim J, Lam M, Chong SA, Keefe RS, Lee J. Unraveling the relationship between obesity, schizophrenia and cognition. Schizophr Res. 2013;151:107-12.

46 Gorczynski P, Faulkner G. Exercise therapy for schizophrenia. Schizophr Bull. 2010;36:665-6.

47 Hodgson M, McCulloch HP, Fox KR. The experiences of people with severe and enduring mental illness engaged in a physical activity programme integrated into the mental health service. Menta Health Phys Act. 2011;4:23-9. 